\title{
What Is the Origin of These Nail Changes in an Otherwise Healthy Young Patient? (From page 31)
}

\author{
Ashley Webb, MS, PharmD, William Kerns II, MD
}

Carolinas Poison Center, Charlotte, NC and Carolinas Medical Center, Charlotte, NC

Answer: The patient developed Mees' lines due to selenium toxicity.

This previously healthy female experienced 4 months of intermittent, nonspecific illness that she initially attributed to the "flu." First symptoms included severe myalgias and arthralgias. Associated symptoms included intermittent nausea, anorexia, fatigue, weight loss, and memory impairment. Two weeks after onset of illness, her hair started falling out in handfuls and her nails became yellow and brittle. Sores developed at the site of scalp hair loss and on her perineum. Throughout her illness, she experienced intermittent hives. Despite several medical evaluations, no etiology was identified. Of note, the patient's previously healthy children (ages 1, 4, and 8 years) also developed intermittent illness characterized by diarrhea, hives, bronchospasm, and mouth blisters.

Selenium was ultimately identified as the etiology following a nationwide recall of a nutritional supplement due to excessive selenium content. The patient and her children regularly consumed Total Body Formula without incident for the previous 3 years. However, she started a new bottle just prior to onset of illness. The intended selenium content was $7.33 \mu \mathrm{g} / \mathrm{ml}$, but the recalled product contained $800.5 \mu \mathrm{g} / \mathrm{ml}$ [1]. The patient's serum selenium level 4 months after onset of illness was $233 \mu \mathrm{g} / \mathrm{L}$ (reference range 110-160). Most symptoms subsided by 30 days following cessation of product consumption, consistent with the half-life of selenium. Her nails improved, but had persistent abnormalities as seen in the image.

Mees' lines are white bands across the entire width of the fingernails. They are most commonly associated with metal toxicity, including arsenic or thallium, but have also been noted in selenosis, carbon monoxide poisoning, chemotherapy, heart failure, Hodgkin's disease, and malaria [2]. Functionally, selenium is essential for production of selenoproteins that participate in several enzymatic reactions, including glutathione peroxidase, a free radical scavenger. When in excess, selenium is thought to disrupt other enzymes and functional proteins by substituting for sulfur. Selenium is chemically characterized as a nonmetal, but clinical manifestations of selenosis resemble heavy metal poisoning, including dermatologic responses such as Mees' lines and alopecia. These effects may result from selenium substituting for sulfur in keratin proteins, leading to loss of disulfide bridges and development of abnormal protein structure. Notably, hair and nails may serve as an excretion route for excess selenium [3].

Other common manifestations of selenosis include nausea, vomiting, diarrhea, weight loss, fatigue, myalgias, arthralgias, peripheral neuropathy, cognitive complaints, and halitosis. Halitosis or garlic breath may result from selenium excretion in expired air. The patient and her children had an unusual manifestation of intermittent urticaria.

In retrospect, the intermittent nature of illness in this case was due to dietary interference. Nausea and anorexia led to cessation of supplement use and resolution of gastrointestinal symptoms. As gastrointestinal effects abated, she restarted the supplement with subsequent return of symptoms. Although selenium levels were within the reference range in her children, their illness was consistent with selenosis. Exposure in the oldest children occurred via direct oral intake. Breastfeeding was responsible in the youngest child [3].

\section{REFERENCES}

1. Sutter M, Thomas JD, Brown J, et. al. Selenium Toxicity: A case of selenosis caused by a nutritional supplement. Ann Intern Med 2008;148:970-971.

2. Fawcett R, Linford S, Stulberg LD. Nail Abnormalities: Clues to Systemic Disease. Am Fam Physician 2004;69:1417-1424.

3. Klaassen C, editor. Casarett and Doull's Toxicology, The Basic Science of Poisons. 7th ed. New York: McGraw Hill Medical, 2008: 957-958.

Keywords: Selenium, Mees' lines, metal toxicity

Note: The authors have no potential financial conflicts of interest to report. 